\title{
NUMERICAL STUDY OF TERAHERTZ PULSE GENERATION FROM FEW-CYCLE LASER PULSES IN THE MID-IR SPECTRAL RANGE
}

\author{
Roland Flender ${ }^{1}$, Adam Borzsonyi ${ }^{1,2}$, Viktor Chikan ${ }^{1,3}$ \\ ${ }^{1}$ ELI-ALPS, ELI-HU Non-Profit Ltd., Wolfgang Sandner utca 3., Szeged, H-6728, Hungary \\ ${ }^{2}$ Dept. of Optics and Quantum Electronics, Univ. of Szeged, Dóm tér 9., H-6720 Szeged, Hungary \\ ${ }^{3}$ Dept. of Chemistry, Kansas State University, 213 CBC Building, Manhattan, KS 66506-0401, USA.
}

DOI: https://doi.org/10.14232/kvantumelektronika.9.10

\section{Introduction}

The terahertz (THz) pulse generation from two-color laser pulse ionized gas is an actively researched topic since its discovery [1]. In this method, the fundamental pulse and its second harmonic pulse are focused in ambient or in different gases. After the tunnel ionization, the free electrons accelerate in the external electric field, the $\mathrm{THz}$ pulse generation is the most efficient, when the electrons accelerate in one direction $[2,3]$. The electrons accelerate in one direction, when the external electric field is asymmetric and this occurs when the relative phase is $\pm \pi / 2 \mathrm{rad}$ between the fundamental pulse and its second harmonic pulse.

Alternatively, one can use few-cycle one-color pulses to generate $\mathrm{THz}$ pulse with the same method. In this case, the external electric field is asymmetric intrinsically, thus it will accelerate the electrons effectively in one direction and generates $\mathrm{THz}$ pulse effectively. The greatest advantage of the onecolor method is simplicity, that there is no need of any nonlinear crystal, therefore there is no reflection loss, no absorption loss and no dispersion. In this study we compared the $\mathrm{THz}$ pulse generation from one- and two-color laser pulses.

\section{Results}

We are particularly interested in THz pulse generation based on mid-infrared (MIR) laser source, since the efficiency is more promising with longer wavelengths compared to conventional nearinfrared lasers. The laser pulse parameters for the numerical simulation are based on the MIR laser system [4, 5] located at ELI-ALPS user facility [6]. The central wavelength of this laser system is $3.2 \mu \mathrm{m}$ and the spectral bandwidth supports $45 \mathrm{fs}$ pulse duration, the pulse energy at the output window is $140 \mu \mathrm{J}$. In our simulation, we changed the pulse duration from 1.0 optical cycle (10.67 fs) to 5.0 optical cycles $(53.33 \mathrm{fs})$, while keeping the peak intensity at a constant value. Our simulation was based on an assumed setup shown in Fig. 1.

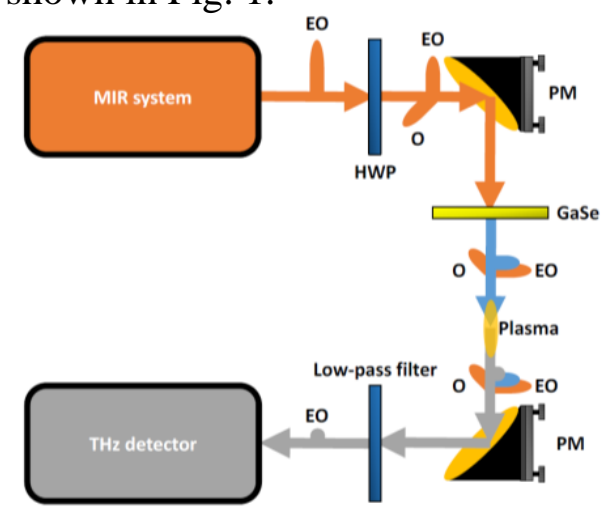

Fig.1 The schematic drawing of a virtual setup used in our simulations. EO-extraordinary polarization, O- ordinary polarization, $H W P$ - half-wave plate, PM-parabolic mirror, GaSe-gallium selenide. 
In this research, we have examined numerically the $\mathrm{THz}$ pulse generation with one- and two-color laser pulses (see Fig. 2.). The dependence on pulse duration and polarization angle have been investigated. By changing the polarization angle the efficiency of the second harmonic generation (SHG) can be changed. The $0^{\circ}$ polarization angle means, that the driving laser pulse polarization is purely extraordinary, hence there is no SHG occurs in the gallium selenide (GaSe) crystal.

In general, we found that in the case of one-color method, the intensity are increasing with decreasing pulse duration (red arrows in both Fig. 2. a and b). The mechanism behind this, that with shorter pulses the external electric field are more asymmetric, which increase the $\mathrm{THz}$ pulse generation efficiency despite the pulses have less energy. In the case of two-color method, the intensity are decreasing with decreasing pulse duration (blue arrows in both Fig. 2. a and b). The mechanism behind this, that the external electric field are already asymmetric and with shorter pulses the pulses have less energy, which decrease the THz pulse generation efficiency.

(a)

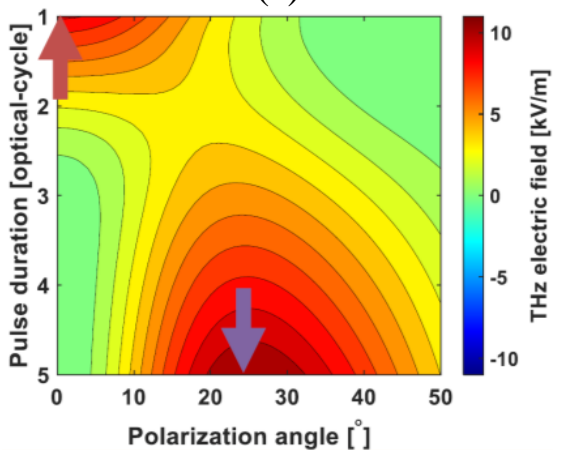

(b)

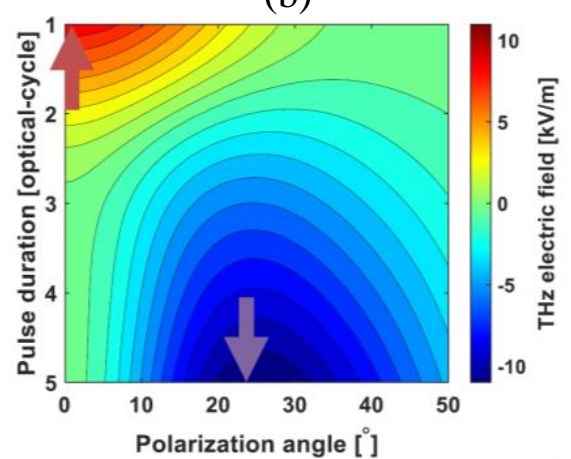

Fig 2. The THz pulse peak power in the function of the pulse duration and the polarization angle in case of a.) $+\pi / 2 \mathrm{rad}$ and b.) $-\pi / 2 \mathrm{rad}$ relative phase. The central wavelength of the driving laser is $3200 \mathrm{~nm}$.

In the case of $+\pi / 2 \mathrm{rad}$ relative phase (see Fig. 2. a), there are two regions, where efficient $\mathrm{THz}$ pulse generation is realized. The first region is the conventional region, where the two-color scheme is the more effective process. This region can be seen in the bottom-middle (blue arrow) on Fig. 2. a. The second region is the unconventional region, where the one-color scheme is the more effective process. This region can be seen in the top-left (red arrow) on Fig. 2. a. The conventional and the unconventional region also can be observed in the case of $-\pi / 2 \mathrm{rad}$ relative phase (see Fig. 2 . b). However, the sign of the conventional region is inverted in this case as one would expect.

Comparing the two cases one can see, there are three regions with different behaviors. The first is the unconventional region, where the single-color $\mathrm{THz}$ pulse generation is more efficient. The second is the conventional region, where the double-color $\mathrm{THz}$ pulse generation is more efficient and furthermore the $\mathrm{THz}$ pulse generation are not sensitive on the sign of the relative phase. The third and final is the semi-conventional region, where the double-color $\mathrm{THz}$ pulse generation is more effective and however the THz pulse generation are sensitive on the sign of the relative phase. This region is between the conventional and unconventional regions.

This behavior is mostly similar to all wavelengths of the studied spectral range. Single-color driving laser pulses are more efficient below two optical cycles. The upper limit of the unconventional region is 1.9 cycles $(\sim 16 \mathrm{fs})$ at $2500 \mathrm{~nm}$ and 1.6 cycles $(\sim 21 \mathrm{fs})$ at $4000 \mathrm{~nm}$. The conventional region starts above three cycles. More precisely 3.2 cycles ( $27 \mathrm{fs})$ at $2500 \mathrm{~nm}$ and 3.5 cycles ( $47 \mathrm{fs})$ at $4000 \mathrm{~nm}$. 

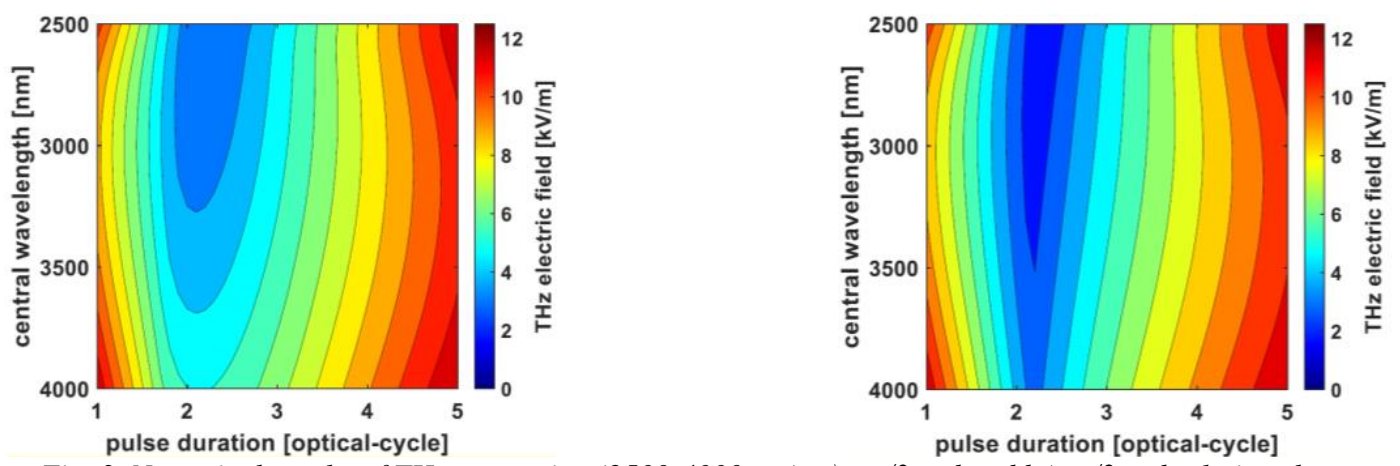

Fig. 3. Numerical results of THz generation $(2500-4000 \mathrm{~nm})$ a.) $+\pi / 2 \mathrm{rad}$ and $\mathrm{b}$.) $-\pi / 2$ rad relative phase.

\section{Summary}

In summary, we have investigated the $\mathrm{THz}$ pulse generation with one- and two-color pulses in the MIR spectral range. In the numerical simulations the pulse duration changed from 1.0 optical cycles (10.67 fs) to 5.0 optical cycles (53.33 fs). We have found that in the case one-color method the $\mathrm{THz}$ pulse spectral components are increasing with decreasing pulse duration, while in the case of twocolor method the $\mathrm{THz}$ pulse spectral components are decreasing with decreasing pulse duration. If the pulse duration is shorter than 1.5 optical cycles then the one-color method is more efficient, else the two-color method is more efficient.

\section{Acknowledgement}

The ELI-ALPS project (GINOP-2.3.6-15-2015-00001) is supported by the European Union and cofinanced by the European Regional Development Fund. The project has been supported by the European Union, co-financed by the European Social Fund. EFOP-3.6.2-16-2017-00005-“Ultrafast physical processes in atoms, molecules, nanostructures and biological systems."

\section{References}

[1] D. J. Cook et al. Optics Letters 25(16), 1210-1212 (2000)

https://doi.org/10.1364/OL.25.001210

[2] Y. Watanabe et al. Opt. Comm. 234, 125-129 (2004)

https://doi.org/10.1016/j.optcom.2004.02.032

[3] J. Dai et al. App. Phys. Lett. 94(2), 021117 (2009)

https://doi.org/10.1063/1.3068501

[4] N. Thiré et al. Opt. Exp. 25(2) 1505-1514 (2017)

https://doi.org/10.1364/OE.25.001505

[5] N. Thiré et al. Opt. Exp. 26(21) 26907-26915 (2018)

https://doi.org/10.1364/OE.26.026907

[6] http://www.eli-alps.hu 